\title{
CFD Simulation of Flow Maldistribution due to Blockage in Microstructured Heat Exchanger
}

\author{
Sahba Sadir*, ${ }^{*}$, Christoph Spiegel ${ }^{2}$, Wolfgang Augustin ${ }^{2}$, Stephan Scholl ${ }^{2}$ and Manfred Kraut ${ }^{1}$ \\ ${ }^{1}$ Karlsruhe Institute of Technology, Institute for Micro Process Engineering \\ Hermann-von-Helmholtz-Platz 1, 76344 Eggenstein-Leopoldshafen, Germany \\ sahba.sadir@kit.edu; manfred.kraut@kit.edu \\ ${ }^{2}$ Technische Universität Braunschweig, Institute for Chemical and Thermal Process Engineering \\ Langer Kamp 7, 38106 Braunschweig, Germany \\ christoph.spiegel@tu-braunschweig.de; w.augustin@tu-baunschweig.de; s.scholl@tu-braunschweig.de
}

\section{Extended Abstract}

Modern technologies intend to use micro-scaled systems in a wide variety of chemical, biological and heat exchange applications to increase reliability and enhance process and heat transfer efficiency [1]. However, on the downside they can suffer from continuous fouling processes or complete blockage of single flow paths [2,3]. Such perturbations inside the fluid domain have a significant impact on flow distribution and continuous mass flow rate in microstructure geometry which is essential for heat exchangers performance [4]. Therefore, before starting the thermal efficiency analysis of a micro heat exchanger, the uniformity of flow distribution in the microstructure geometry must be assessed.

Microstructured heat exchangers typically comprise a number of parallel microchannels with inlet and outlet headers. The uniformity of flow rates among the parallel microchannels is governed by the field of fluid pressure in the system. Computational fluid dynamic (CFD) is generally used to characterize the fluid flow in microstructures and manifolds and provides proper recommendations for designing micro heat exchangers.

In this contribution, three dimensional (3D) CFD simulations are performed using OpenFOAM (Open Source Field Operation and Manipulation) by employing simpleFOAM solver to investigate flow distribution in an array of four parallel microchannels with a hydraulic diameter of $500 \mu \mathrm{m}$ each. The working fluid is water and an incompressible and singlephase flow is assumed. The maldistribution is induced by a man-made blockage of single microchannels in different scenarios. Both numbers of blocked microchannels and the position of the blockage are altered. The results show the effect of induced blockage on pressure drop and fluid flow distribution in the parallel microchannels. The pressure drop significantly increases by increasing the number of blocked channels as is to be expected. The standard deviation of flow distribution not only relies on the total number of the blocked microchannels but also on their position inside the flow array. For validating CFD results, pressure drop measurements for different scenarios are conducted. A good agreement between simulation and experiment is observed. Finally, the influence of the maldistribution on the heat exchanger performance is presented and discussed.

\section{References}

[1] O. Brand, G.K. Fedder, C. Hierold, J.G. Korvink and O. Tabata, Micro process engineering: fundamentals, devices, fabrication, and applications. John Wiley \& Sons, 2013.

[2] M. Schoenitz, L. Grundemann, W. Augustin, and Scholl, Fouling in microstructured devices: a review. Chemical Communications, 2015, 51(39), pp.8213-8228.

[3] C. Spiegel, M. Kraut, G. Rabsch, C. Küsters, W. Augustin, and S. Scholl, Fouling Detection in an Optically Accessible Microstructured Heat Exchanger. Chemical Engineering \& Technology, 2019, 42(10), pp.2067-2075.

[4] E.V. Rebrov, J.C. Schouten and M.H. De Croon, Single-phase fluid flow distribution and heat transfer in microstructured reactors. Chemical Engineering Science, 2011, 66(7), pp.1374-1393. 